\title{
Modelling and simulation of a multi-phase traffic light controlled T-type junction using timed coloured petri nets
}

\author{
*Ganiyu R. A., Olabiyisi S. O., Omidiora E. O., Okediran O. O. and Alo O. O. \\ Department of Computer Science and Engineering, \\ Ladoke Akintola University of Technology, P.M.B 4000, Ogbomoso, Nigeria. \\ ${ }^{*}$ Corresponding author
}

\begin{abstract}
The emergence of traffic and subsequently traffic congestion in urban road networks are increasing worldwide with the growing number of vehicles. In the worst case, traffic congestion results in excess delays, reduced safety and increased environmental pollution. As a result, to proffer effective solutions to the aforementioned congestion problems, a vast amount of literature offered fixed-time or traffic-response control strategies, each focusing on modelling traffic light controlled intersections. In this paper, a Timed Coloured Petri Net (TCPN) formalism is employed to model and simulate a multi-phase traffic light controlled intersection with an associated fixed signal timing plan using T-type junction located in Federal Capital Territory, Abuja, Nigeria, as a case study. Besides, the developed model is simulated via CPN tools while its validation is carried out on the basis of real traffic data measured by detectors positioned at each input link of the T-type junction under consideration.
\end{abstract}

Keywords: Timed Coloured Petri Net, Traffic light, T-type Junction, Stream

\section{INTRODUCTION}

The rapid growth of urban traffic requires efficient control methods. Traffic congestion appears when too many vehicles attempt to use a common transportation infrastructure with limited capacity. It leads to a degraded use of the available infrastructure, thus contributing to an accelerated congestion increase, which leads to further infrastructure degradation, and so forth. However, to keep traffic flowing smoothly and safely, traffic lights at intersections is the major control measure in urban road networks. Among the main advantages of traffic signals are the flexibility of the signaling scheme, the ability to provide priority treatment and the feasibility of coordinated control along streets. Nevertheless, when not well designed, traffic signals may lead to excessive delays when cycle lengths are too long and increase the risk of collisions.

The control of a single intersection (belonging to the class of road traffic control) is usually based on a fixed-time strategy or a traffic-response strategy (Kutil et al., 2006). In a fixed-time strategy, the light control phases (i.e. the duration of green and red light) are scheduled offline. The light control phases are derived from historical data measured in a given intersection. There are typically several light control phases for each intersection, depending on the given time of the day. It is equally important to notice that the fixed-time strategy cannot respond to any change in traffic condition since their settings are based on historical data rather than real-time data. On the other hand, traffic-response strategy is based on feedback from the current state of traffic. It receives real-time data through sensors and creates an optimal timing plan. In a real-time control strategy, detectors located on the intersection approaches monitor traffic conditions and feed information on the actual system state to the real-time controller. Moreover, the controller selects the duration of the green phases in the signal-timing plan in order to optimize a performance index (Patel and Ranganathan, 2001; Wey, 2000; Dotoli et al., 2003). In both control strategies, the traffic network has to be appropriately modelled, either for simulation purposes or in order to determine on line some states of the transportation network that are not available due to detector absence or failures (Gabard, 1991).

Nevertheless, one way to approach the challenge of developing concurrent systems is to build an executable model of the system. Constructing a model and simulating it usually lead to significant new insights into the design and operation of the system considered and often results in a simpler and more 
streamlined design. Petri nets have been proven to be a powerful modeling tool for various kinds of discrete event systems (Murata, 1989; Peterson, 1981), and its formalism provides a clear means for presenting simulation and control logic. Hence, the Petri nets are applied in traffic control. Coloured Petri net (CPN) is a graphical oriented language for modelling and validation of systems in which concurrency, communication, and synchronization play a major role. It is a discrete-event modeling language combining the classical Petri nets with the functional programming language Standard ML. More specifically, Petri nets provide the primitives for describing synchronization of concurrent processes, while programming languages provide the primitives for definition of data types and manipulation of their data values (Jensen et al., 2007).

A Coloured Petri Net model of a system is an executable model representing the states of the system as well as the events that can cause the system to change state. The inclusion of time concepts into the Coloured Petri Net model results in a model called Timed Coloured Petri Net (TCPN) model (Ganiyu et al., 2011). In a Timed Coloured Petri Net model, a global clock models the passage of time and through this, it would be possible to calculate performance measures, such as the speed by which a system operates and throughput. Besides, TCPN models may be investigated by means of simulation and validated by a number of mathematical analysis techniques, such as state graphs and invariants. CPN Tools is an industrialstrength computer tool for constructing and analyzing TCPN models.

\section{METHODOLOGY}

Basic Definitions of Coloured Petri Net and Timed Coloured Petri Net: In developing the proposed model, the following basic definitions of Coloured Petri Net (CPN) and Timed Coloured Petri Net (TCPN) were employed:

In a formal way, a Coloured Petri Net is a tuple CPN $=(\Sigma, P, T, A, N, C, G, E, I)$ where:

(i) $\quad \Sigma$ is a finite set of non-empty types, also called colour sets.

(ii) $\mathrm{P}$ is a finite set of places.

(iii) $\mathrm{T}$ is a finite set of transitions.

(iv) $A$ is a finite set of arcs such that:

$P \cap T=P \cap A=T \cap A=\varnothing$. (v) $\quad \mathrm{N}$ is a node function. It is defined from $\mathrm{A}$ into PXT $\cup T X P$.

(vi) $\quad \mathrm{C}$ is a colour function. It is defined from $\mathrm{P}$ into $\Sigma$.

(vii) $\quad \mathrm{G}$ is a guard function. It is defined from $\mathrm{T}$ into expressions such that:

$\forall t \in T:[\operatorname{Type}(\mathrm{G}(\mathrm{t})) \quad=\mathrm{B} \quad$ Type $(\operatorname{Var}(\mathrm{G}(\mathrm{t})))$ $\subseteq \Sigma]$

(viii) $\mathrm{E}$ is an arc expression function. It is defined from A into expressions such that:

$\forall a \in A: \quad\left[\operatorname{Type}(\mathrm{E}(\mathrm{a})) \quad=\quad \mathrm{C}(\mathrm{p})_{\mathrm{MS}}\right.$ $\operatorname{Type}(\operatorname{Var}(E(a))) \subseteq \Sigma$ ] where $p$ is the place of $\mathrm{N}(\mathrm{a})$.

(ix) I is an initialization function. It is defined from $P$ into closed expressions such that:

$\forall p \in P:\left[\right.$ Type $\left.(\mathrm{I}(\mathrm{p}))=\mathrm{C}(\mathrm{p})_{\mathrm{MS}}\right]$.

(Jensen, 1993 and 1994)

A timed non-hierarchical Coloured Petri Net is a tuple $\mathrm{TCPN}=\left(\mathrm{CPN}, \mathrm{R}, \mathrm{r}_{\mathrm{o}}\right)$ such that:

(i) CPN satisfying the above definition.

(ii) $\mathrm{R}$ is a set of time values, also called time stamps. It is closed under + and including 0 .

(iii) $\quad r_{0}$ is an element of $R$ called the start time.

(Huang and Chung, 2008)

\section{Elements of Coloured Petri Net (CPN)}

- $\quad$ The ellipses and the circles are called places. They describe the states of the system.

- The rectangles are called transitions. They describe the actions (processes).

- The arrows are called arcs. The arc expressions describe how the state of the Coloured Petri Net changes when the transitions occur.

- Each place can be marked with one or more tokens, and each token has a data value (token colour) attached it.

Traffic Lights at Junctions: Traffic light signals are designed to keep traffic flowing smoothly and safely. The traffic light signals are meant to be obeyed at junctions by road users. However, the traffic lights of a generic traffic network are defined according to a signal timing plan, including green, red and yellow 
(amber) signals that in Nigerian cities (where available) correspond respectively to you may go on if the way is clear, stop and stop at the stop line.

Timing Plan Decision Variables: A signal timing plan controls vehicle stream in an intersection of predetermined time. Among the main decision variables in a timing plan are cycle time and green splits. Cycle time is defined as the duration of time from the centre of the red phase to the centre of the next red phase. Green split for a signal in a given direction of movement is defined as the fraction of cycle time when the light is green in that direction. Moreover, a phase is the time interval during which a given combination of traffic signals in the area is unchanged. Finally, during each phase different streams may be allowed to proceed, where a stream of vehicles in a junction is a portion of traffic formed by all vehicles that cross the intersection from the same departure link and are directed to the same arrival link in the considered phase.

The Case Study: Figure 1.1 depicts the snapshot of a multi-phase traffic light controlled T-type junction under consideration. The junction is located in Federal Capital Territory, Abuja, Nigeria. Traffic is currently ruled in the junction by a fixed time control strategy with an associated signal-timing plan. Besides, the intersection is regularly crossed by cars, trucks, public transportation buses and mopeds. Figure 1.2 also shows the layout of the T-type junction. The layout consists of two roads named Herbert Macaulay Way and Ladi Kwali Street. This corresponds to ARTERIAL N5 and HOTEL COLLECTOR, respectively, in Figure 1.2. With respect to the three input links of the intersection, the direction from ARTERIAL N5 is identified as East while its opposite direction identified as West. Similarly, the direction from HOTEL COLLECTOR is identified as North.

Furthermore, Table 1.1 describes the input links, the number of lanes, stream identifications and streams associated with the layout of the T-type junction shown in Figure 1.2. Also, Table 1.2 reports the fixed signal-timing plan that is currently implemented in the T-type junction. In particular, the streams allowed to proceed during the phases of the signal timing plan in Table 1.2 are depicted in Figure 1.3 and labelled with letters corresponding to those indicated in Figure 1.2 and Table 1.2. More precisely, vehicle streams are represented with letters from $A$ to $E$, while pedestrian streams are omitted. The considered fixed timing plan comprises 15 phases as shown in Figure 1.3.

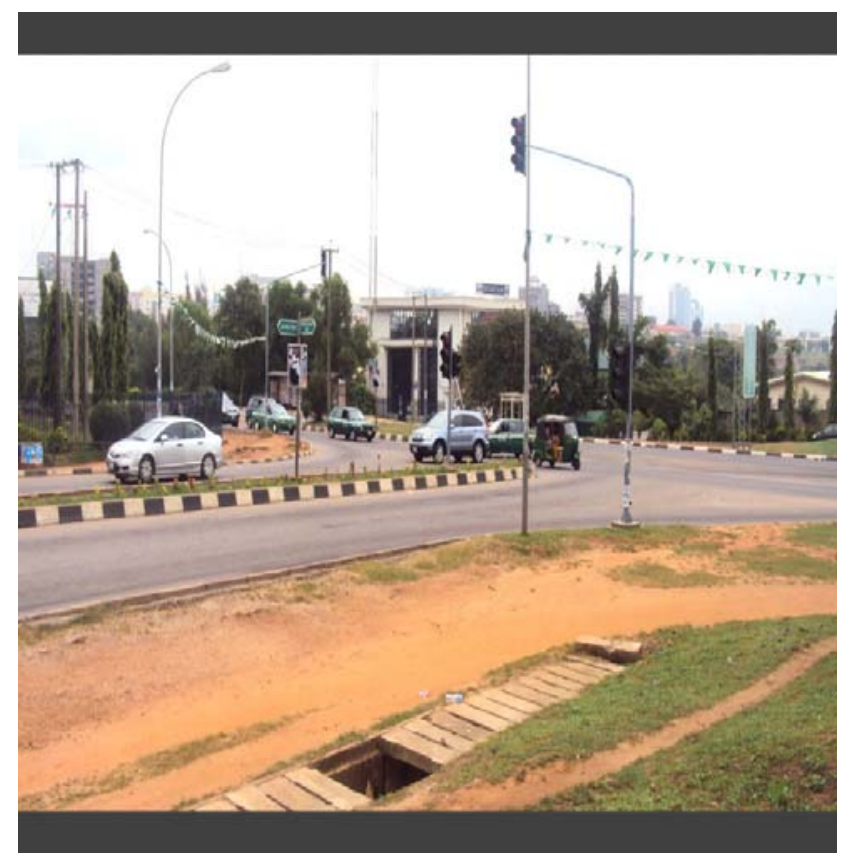

Fig 1.1: Snapshot of a T-type junction located in FCT, Abuja, Nigeria.

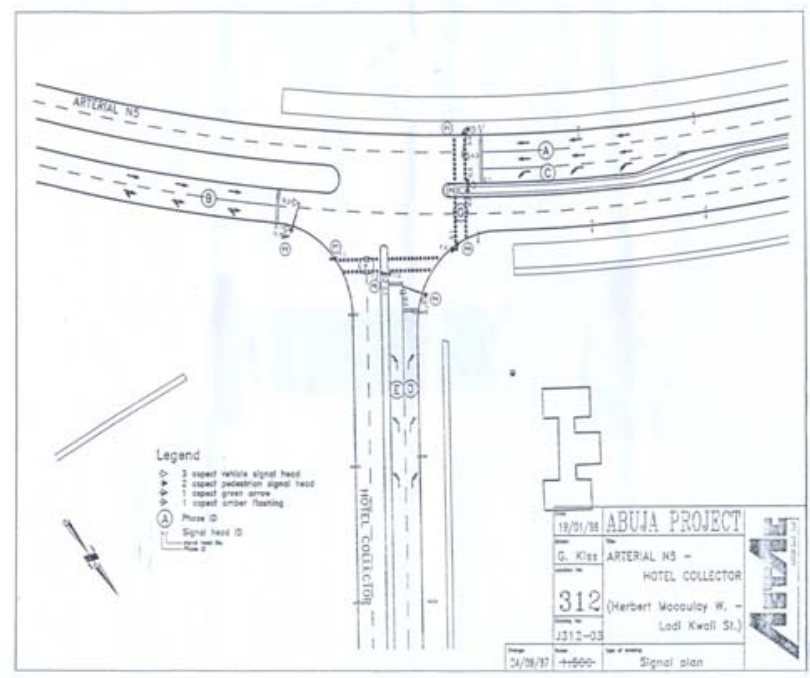

Fig 1.2: Layout of the T-type junction depicted in Figure 1.1 
Table 1.1: The Stream Identifications of the T-type junction

\begin{tabular}{|c|c|c|c|c|}
\hline Input links & $\begin{array}{c}\text { Stream } \\
\text { Identification }\end{array}$ & Stream & Number of Lane & $\begin{array}{l}\text { Traffic Light } \\
\text { Dedicated }\end{array}$ \\
\hline \multirow{2}{*}{$\begin{array}{c}\text { Herbert Macaulay Way } \\
\text { (ARTERIAL N5) from } \\
\text { West }\end{array}$} & A & $\begin{array}{c}\text { A-through } \\
\left(\mathrm{A}_{T}\right)\end{array}$ & 2 & $\begin{array}{l}\text { Two sets of traffic lights } \\
\text { placed on a long arm } \\
\text { cantilever (i.e. A.1 and } \\
\text { A.2) }\end{array}$ \\
\hline & C & $\begin{array}{l}\text { C-left } \\
\left(C_{L}\right)\end{array}$ & 1 & $\begin{array}{l}\text { A set of traffic light placed } \\
\text { on a short arm cantilever } \\
\text { (i.e. C.1) }\end{array}$ \\
\hline \multirow{2}{*}{$\begin{array}{c}\text { Herbert Macaulay Way } \\
\text { (ARTERIAL N5) from } \\
\text { East }\end{array}$} & \multirow[b]{2}{*}{ B } & $\begin{array}{c}\text { B-through } \\
\left(\mathrm{B}_{\mathrm{T}}\right)\end{array}$ & $\begin{array}{c}2 \\
\text { (one dedicated; one } \\
\text { shared with H-right) }\end{array}$ & $\begin{array}{l}\text { Two sets of traffic lights } \\
\text { placed on a long arm } \\
\text { cantilever (i.e. B.1 and } \\
\text { B.2) }\end{array}$ \\
\hline & & $\begin{array}{c}\text { H-right } \\
\left(\mathrm{H}_{\mathrm{R}}\right)\end{array}$ & 1 & $\begin{array}{l}\text { A right turn green arrow } \\
\text { light (i.e. H.1) attached to } \\
\text { a long arm cantilever }\end{array}$ \\
\hline \multirow[t]{2}{*}{$\begin{array}{l}\text { Ladi Kwali Street } \\
\text { (HOTEL COLLECTOR) } \\
\text { from North }\end{array}$} & D & $\begin{array}{c}\text { D-right } \\
\left(D_{R}\right)\end{array}$ & 1 & $\begin{array}{l}\text { A set of traffic light placed } \\
\text { on a long arm cantilever } \\
\text { (i.e. D.1) }\end{array}$ \\
\hline & E & $\begin{array}{l}\text { E-left } \\
\left(E_{L}\right)\end{array}$ & 1 & $\begin{array}{l}\text { A set of traffic light placed } \\
\text { on a short arm cantilever } \\
\text { (i.e. E.1) }\end{array}$ \\
\hline
\end{tabular}

Table 1.2: Signal timing plan of the T-type junction

\begin{tabular}{|c|c|c|c|c|c|c|c|c|c|c|c|c|c|c|c|c|}
\hline \multirow{2}{*}{ Streams } & \multirow{2}{*}{$\begin{array}{l}\text { Signal } \\
\text { Heads }\end{array}$} & \multicolumn{15}{|c|}{ Phases } \\
\hline & & 1 & 2 & 3 & 4 & 5 & 6 & 7 & 8 & 9 & 10 & 11 & 12 & 13 & 14 & 15 \\
\hline$A_{T}$ & A.1 A.2 & $G$ & G & $\bar{Y}$ & $\bar{Y}$ & $\mathrm{R}$ & $\mathrm{R}$ & $R$ & $R$ & $R$ & $\mathrm{R}$ & $\mathrm{G}$ & $\mathrm{G}$ & $G$ & $G$ & $\mathrm{G}$ \\
\hline $\mathrm{B}_{\mathrm{T}}$ & B.1 B.2 & G & $Y$ & $\mathrm{Y}$ & $\mathrm{R}$ & $\mathrm{R}$ & $\mathrm{R}$ & $\mathrm{R}$ & $R$ & $\mathrm{R}$ & $\mathrm{R}$ & $\mathrm{R}$ & $\mathrm{R}$ & $\mathrm{R}$ & $\mathrm{R}$ & G \\
\hline $\mathrm{C}_{\mathrm{L}}$ & C.1 & $\mathrm{R}$ & $\mathrm{R}$ & $\mathrm{R}$ & $\mathrm{R}$ & $\mathrm{R}$ & $\mathrm{R}$ & $\mathrm{R}$ & $\bar{R}$ & $\mathrm{R}$ & $\mathrm{R}$ & $G$ & $\mathrm{Y}$ & $\mathrm{R}$ & $\mathrm{R}$ & $\mathrm{R}$ \\
\hline$D_{R}$ & D.1 & $\mathrm{R}$ & $\mathrm{R}$ & $\mathrm{R}$ & $\mathrm{R}$ & $\mathrm{R}$ & $\mathrm{R}$ & $G$ & G & $G$ & $G$ & $G$ & $G$ & $\mathrm{Y}$ & $\mathrm{R}$ & $\mathrm{R}$ \\
\hline$E_{L}$ & E.1 & $\mathrm{R}$ & $\mathrm{R}$ & $\mathrm{R}$ & $\mathrm{R}$ & $\mathrm{R}$ & G & $G$ & G & $\mathrm{Y}$ & $\mathrm{R}$ & $\mathrm{R}$ & $\mathrm{R}$ & $\mathrm{R}$ & $\mathrm{R}$ & $\mathrm{R}$ \\
\hline $\mathrm{H}_{\mathrm{R}}$ & H.1 & & G & G & G & G & G & G & & & & & & & & \\
\hline \multicolumn{2}{|c|}{ Phase Duration(sec) } & 37 & 1 & 2 & 1 & 3 & 1 & 17 & 1 & 3 & 2 & 20 & 3 & 3 & 1 & 5 \\
\hline \multicolumn{2}{|c|}{$\begin{array}{l}\text { Cycle } \\
\text { Duration(sec) }\end{array}$} & \multicolumn{15}{|c|}{100} \\
\hline
\end{tabular}
Legend
G: Green signal
Y: Yellow signal
R: Red signal 


\section{Modelling the T-type Junction:}

The proposed Timed Coloured Petri Net (TCPN) model of the T-type junction under consideration is developed consisting of two parts, namely the intersection sub-model and the traffic light submodels. Based on the Timed Coloured Petri Net formalism, the tokens required for both intersection and traffic light sub-models comprise three elements. The token elements (i.e. $i, t$ and $n$ ) and their interpretations are as enumerated:

- The element $i$ denotes the incoming vehicles into an intersection (i.e. stream $A_{T}, B_{T}, C_{L}$, $D_{R}, E_{L}, H_{R}$ vehicles).

- The element $t$ represents the average time interval of vehicles entering an intersection with respect to a particular stream during green phase of each cycle.

- $\quad$ The element $n$ represents a counter counting the number of vehicles entering or leaving an intersection.

However, the parameters necessary to describe the traffic behaviours in the T-type junction are as follows:

- The number of vehicles entering the intersection during green duration of each stream is measured by detectors (i.e. traffic data counters) positioned at each input link of the T-type intersection.

- The phase durations (in seconds) of green, red and yellow signal lights of each stream as reported in signal timing plan shown in Table 1.2.

The Intersection Sub-model of the T-type Junction: Traffic light signals are an important mechanism applied to solve intersection conflicts and regulate traffic flow. To keep traffic flowing smoothly and safely, the intersection part of the proposed TCPN model is designed to model the vehicles passing through the T-type junction under consideration, following the fixed signal timing plan depicted in Table 1.2. In particular, vehicles are allowed to enter the intersection through a particular input link when the signal light controlling the input link turns green.

However, the Intersection Sub-model requires four major places (to be drawn as circles and named Avgt, DB, $V r$, and $V i_{i}$ ), two transitions (to be drawn as rectangular boxes and named Movi and Movo, a number of directed arcs connecting places and transitions, and finally some textual inscriptions next to the places (i.e. NI and IT) and arcs (e.g. (n, i) and $(i, t)$ ). By considering the firing sequence of the two transitions (Movi and Movo), the presence of tokens in the aforementioned places are defined as follows:

- A token in place Avgt represents the average time interval of vehicles entering the intersection with respect to a particular stream.

- A token in place $V r$ means a vehicle ready for entering the intersection.

- Place Vi with a token means a vehicle passing through the intersection.

- Tokens in place DB represents outgoing vehicles departing the intersection.

Nevertheless, the transition Movi moves an incoming vehicle into the intersection

The Traffic Light Sub-models of the T-type Junction: To correctly control an intersection via traffic light signal indications, each traffic signal must follow a defined sequence of active colour lights, normally from green to yellow and red, and then backing to green. As a result, the traffic light part of the proposed TCPN model modelled the changing rule of traffic lights according to the fixed signaltiming plan shown in Table 1.2. In particular, vehicles are allowed to pass through an intersection when green lights are turned on. On the other hand, vehicles are inhibited to pass through an intersection during red and yellow signal indications as these, in Nigerian context, correspond to stop and stop at the stop line, respectively.

By considering the T-type junction shown in Figure 1.2, there are six vehicle streams identified, namely C-left, D-right, E-left, B-right, A-through and Bthrough denoted by $C_{L}, D_{R}, E_{L}, H_{R}, A_{T}$ and $B_{T}$, respectively, in Table 1.1. As a result, the traffic light part of the TCPN modelling the T-type junction is divided into six sub-models. These are called streams $C_{L}, D_{R}, E_{L}, H_{R}, A_{T}$ and $B_{T}$ sub-models. As indicated in Table 1.1, each of the first three streams (i.e. $C_{L}, D_{R}$ and $E_{L}$ ) is controlled by one set of traffic light while the next stream (i.e. $H_{R}$ ) is controlled only by a Right turn green arrow light.

Nevertheless, the last two streams (i.e. $A_{T}$ and $B_{T}$ ) are individually controlled by two sets of traffic lights. Precisely, each of the last two streams is allocated two lanes and controlled correspondingly by two sets 
of traffic lights placed on a long arm cantilever. As an example, two sets of traffic lights represented by A.1 and A.2 in Figure 1.2 concurrently control the Stream $A_{T}$ vehicles. As reflected by signal heads depicted in Table 1.2, in modelling stream $A_{T}$ traffic lights, the two sets of traffic lights are merged and represented as follows:

- Place $A_{G}$ models the state of green light controlling the Stream $A_{T}$ vehicles

- Place $A_{Y}$ models the state of yellow light controlling the Stream $A_{T}$ vehicles

- Place $A_{R}$ models the state of red light controlling the Stream $A_{T}$ vehicles

This is also applicable to the two sets of traffic lights ruling the stream $B_{T}$. That is, the two sets of traffic lights controlling the stream $\mathrm{B}_{\mathrm{T}}$ are merged and modelled as stated in Table 1.3. Besides, the model representations of the other traffic lights (i.e. streams $C_{L}, D_{R}, E_{L}$ and $H_{R}$ traffic lights) are also explicated in Table 1.3.

Moreover, taking model representation of the stream $A_{T}$ traffic lights as an example, the presence of token in each of the places $A_{G}, A_{Y}$ and $A_{R}$ means green, yellow and red signal lights turn on respectively, and turn off otherwise. This is also applicable for each of the streams $C_{L}, D_{R}, E_{L}, H_{R}$ and $B_{T}$ traffic lights.

Furthermore, for each of the six traffic light submodels, the transitions required and their functions are enumerated in Table 1.4. Based on the rules of Timed Colored Petri Nets, all the transitions would be drawn as rectangles. These represent individual events taking place in the traffic lights of the T-type intersection. As with places, the names of the transitions are written inside the rectangles (e.g. T1, T2, T3 etc). A number of directed arcs connecting places and transitions are associated with appropriate arc expressions which consist of one or two of the following decision variables:
Table 1.3: Model Representation of Major Traffic Light States of the T-type Junction

\begin{tabular}{|c|c|c|}
\hline $\begin{array}{c}\text { Sub- } \\
\text { models }\end{array}$ & Places & Model Representations \\
\hline \multirow{3}{*}{$\begin{array}{c}\text { Stream } \\
\mathrm{B}_{\mathrm{T}}\end{array}$} & $\mathrm{B}_{\mathrm{G}}$ & $\begin{array}{l}\text { Models the state of green light } \\
\text { controlling the Stream } \\
\text { vehicles. }\end{array}$ \\
\hline & $\mathrm{B}_{Y}$ & $\begin{array}{l}\text { Models the state of yellow light } \\
\text { controlling the Stream } \\
\text { vehicles. }\end{array}$ \\
\hline & $\mathrm{B}_{\mathrm{R}}$ & $\begin{array}{l}\text { Models the state of red light } \\
\text { controlling the Stream } \\
\text { vehicles. }\end{array}$ \\
\hline \multirow{3}{*}{$\begin{array}{c}\text { Stream } \\
C_{L}\end{array}$} & $\mathrm{C}_{\mathrm{G}}$ & $\begin{array}{l}\text { Models the state of green light } \\
\text { controlling the Stream } \\
\text { vehicles. }\end{array}$ \\
\hline & $\mathrm{C}_{Y}$ & $\begin{array}{l}\text { Models the state of yellow light } \\
\text { controlling the Stream } \\
\text { vehicles. }\end{array}$ \\
\hline & $\mathrm{C}_{\mathrm{R}}$ & $\begin{array}{l}\text { Models the state of red light } \\
\text { controlling the Stream } C_{L} \\
\text { vehicles. }\end{array}$ \\
\hline \multirow{3}{*}{$\begin{array}{c}\text { Stream } \\
\mathrm{D}_{\mathrm{R}}\end{array}$} & $D_{G}$ & $\begin{array}{l}\text { Models the state of green light } \\
\text { controlling the Stream } D_{R} \\
\text { vehicles. }\end{array}$ \\
\hline & DY & $\begin{array}{l}\text { Models the state of yellow light } \\
\text { controlling the Stream } D_{R} \\
\text { vehicles. }\end{array}$ \\
\hline & $D_{R}$ & $\begin{array}{l}\text { Models the state of red light } \\
\text { controlling the Stream } D_{R} \\
\text { vehicles. }\end{array}$ \\
\hline \multirow{3}{*}{ Stream $E_{L}$} & $E_{G}$ & $\begin{array}{l}\text { Models the state of green light } \\
\text { controlling the Stream } \\
\text { vehicles. }\end{array}$ \\
\hline & $\mathrm{E}_{\mathrm{Y}}$ & $\begin{array}{l}\text { Models the state of yellow light } \\
\text { controlling the Stream } \\
\text { vehicles. }\end{array}$ \\
\hline & $E_{R}$ & $\begin{array}{l}\text { Models the state of red light } \\
\text { controlling the Stream } E_{L} \\
\text { vehicles. }\end{array}$ \\
\hline $\begin{array}{c}\text { Stream } \\
H_{R}\end{array}$ & $\mathrm{H}_{\mathrm{G}}$ & $\begin{array}{l}\text { Models the state of a Right turn } \\
\text { green arrow light controlling the } \\
\text { stream } H_{R} \text { vehicles. }\end{array}$ \\
\hline
\end{tabular}

- Element $i$ denoting the incoming vehicles into the T-type intersection (i.e. incoming vehicles along streams $A_{T}, B_{T}, C_{L}, D_{R}, E_{L}$ and $H_{R}$ would to be denoted by $i_{a}, i_{b}, i_{c}, i_{d}, i_{e}$ and $i_{h}$ respectively). 
- Element $n$ denoting a counter that counts the number of vehicles entering or leaving the $\mathrm{T}$ type intersection.

- Time stamp derived from durations of green, red and yellow signal lights of each stream shown in Table 1.2. This would be introduced using the symbol @. It should be noted that the time stamp could be defined as seconds, microseconds, etc. Here, one time unit is assumed to be one millisecond.

Table 1.4: Major Transitions of the Six Traffic Light Sub-models

\begin{tabular}{|c|c|c|}
\hline Sub-models & Transitions & Actions \\
\hline \multirow{3}{*}{ Stream $A_{T}$} & $\mathrm{~T}_{17}$ & $\begin{array}{l}\text { Concurrently turns off and on red and green signal lights respectively for Stream } A_{T} \\
\text { vehicles in the first instance. }\end{array}$ \\
\hline & $\mathrm{T}_{21}$ & $\begin{array}{l}\text { Concurrently turns off and on red and green signal lights respectively for Stream } A_{T} \\
\text { vehicles in the second instance. }\end{array}$ \\
\hline & $\mathrm{T}_{18}$ & $\begin{array}{l}\text { Concurrently turns off and on green and yellow signal lights respectively for Stream } A_{T} \\
\text { vehicles. }\end{array}$ \\
\hline \multirow{4}{*}{ Stream $\mathrm{B}_{\mathrm{T}}$} & $\mathrm{T}_{1}$ & $\begin{array}{l}\text { Concurrently turns off and on red and green signal lights respectively for Stream } B_{T} \\
\text { vehicles in the first instance. }\end{array}$ \\
\hline & $\mathrm{T}_{5}$ & $\begin{array}{l}\text { Concurrently turns off and on red and green signal lights respectively for Stream } B_{T} \\
\text { vehicles in the second instance. }\end{array}$ \\
\hline & $\mathrm{T}_{2}$ & $\begin{array}{l}\text { Concurrently turns off and on green and yellow signal lights respectively for Stream } B_{T} \\
\text { vehicles. }\end{array}$ \\
\hline & $\mathrm{T}_{3}$ & $\begin{array}{l}\text { Concurrently turns off and on yellow and red signal lights respectively for Stream } B_{T} \\
\text { vehicles. }\end{array}$ \\
\hline \multirow{3}{*}{ Stream $C_{L}$} & $\mathrm{~T}_{23}$ & $\begin{array}{l}\text { Concurrently turns off and on red and green signal lights respectively for Stream } \mathrm{C}_{\mathrm{L}} \\
\text { vehicles. }\end{array}$ \\
\hline & $\mathrm{T}_{24}$ & $\begin{array}{l}\text { Concurrently turns off and on green and yellow signal lights respectively for Stream } C_{L} \\
\text { vehicles. }\end{array}$ \\
\hline & $\mathrm{T}_{25}$ & $\begin{array}{l}\text { Concurrently turns off and on yellow and red signal lights respectively for Stream } C_{L} \\
\text { vehicles. }\end{array}$ \\
\hline \multirow[b]{2}{*}{ Stream $\mathrm{H}_{\mathrm{R}}$} & $\mathrm{T}_{7}$ & Turns on a Right turn green arrow light for Stream $\mathrm{H}_{\mathrm{R}}$ vehicles. \\
\hline & $\mathrm{T}_{8}$ & Turns off a Right turn green arrow light for Stream $\mathrm{H}_{\mathrm{R}}$ vehicles \\
\hline \multirow{3}{*}{ Stream $D_{R}$} & $\mathrm{~T}_{9}$ & $\begin{array}{l}\text { Concurrently turns off and on red and green signal lights respectively for Stream } D_{R} \\
\text { vehicles. }\end{array}$ \\
\hline & $\mathrm{T}_{10}$ & $\begin{array}{l}\text { Concurrently turns off and on green and yellow signal lights respectively for Stream } D_{R} \\
\text { vehicles. }\end{array}$ \\
\hline & $\mathrm{T}_{11}$ & $\begin{array}{l}\text { Concurrently turns off and on yellow and red signal lights respectively for Stream } D_{R} \\
\text { vehicles. }\end{array}$ \\
\hline \multirow{3}{*}{ Stream $\mathrm{E}_{\mathrm{L}}$} & $\mathrm{T}_{13}$ & $\begin{array}{l}\text { Concurrently turns off and on red and green signal lights respectively for Stream } \mathrm{E}_{\mathrm{L}} \\
\text { vehicles. }\end{array}$ \\
\hline & $\mathrm{T}_{14}$ & $\begin{array}{l}\text { Concurrently turns off and on green and yellow signal lights respectively for Stream } \mathrm{E}_{\mathrm{L}} \\
\text { vehicles. }\end{array}$ \\
\hline & $\mathrm{T}_{15}$ & $\begin{array}{l}\text { Concurrently turns off and on yellow and red signal lights respectively for Stream } E_{L} \\
\text { vehicles. }\end{array}$ \\
\hline
\end{tabular}


The Timed Coloured Petri Net Model of the T-type Junction: Figures 1.4 and 1.5 respectively show upper and lower parts of the developed TCPN model of the T-type junction using Timed Coloured Petri Nets. The combination of the two parts (Figures 1.4 and 1.5) gives the overall Timed Coloured Petri Net Model of the T-type junction.

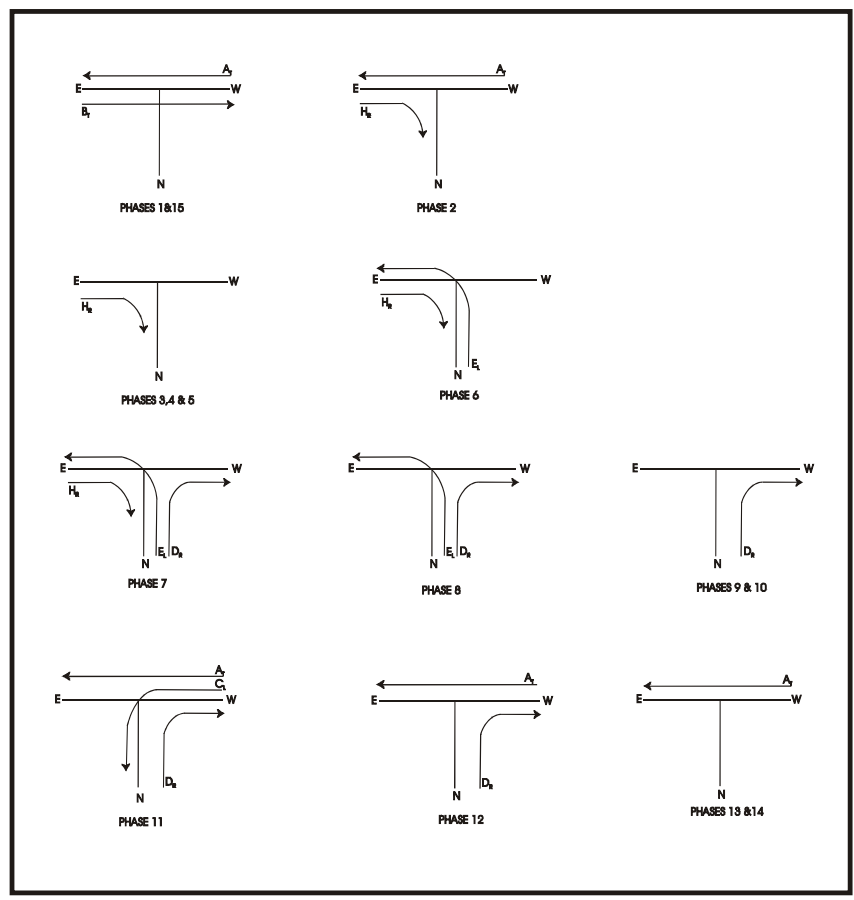

Fig 1.3: Vehicle streams in each phase of the signal timing plan of the T-type junction.

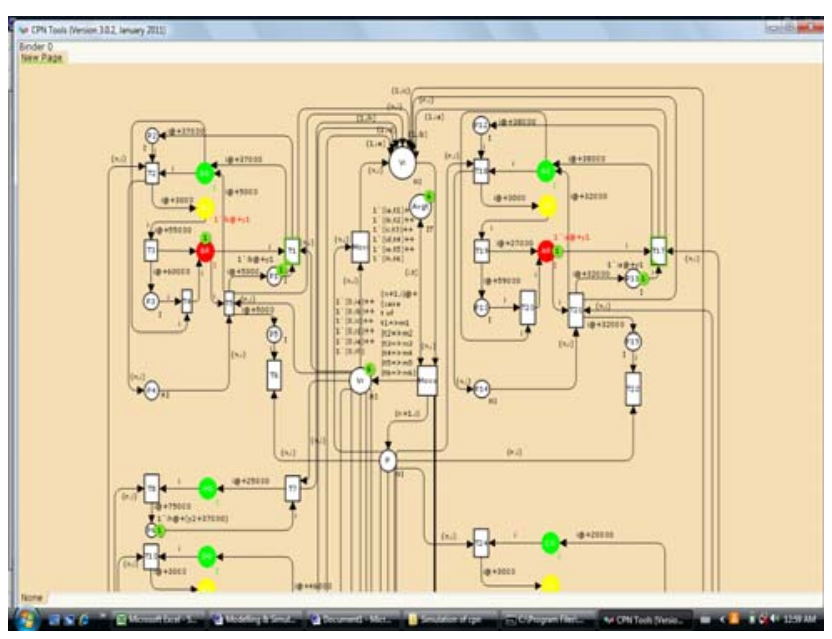

Fig 1.4: Upper part of the developed TCPN model of the T-type junction

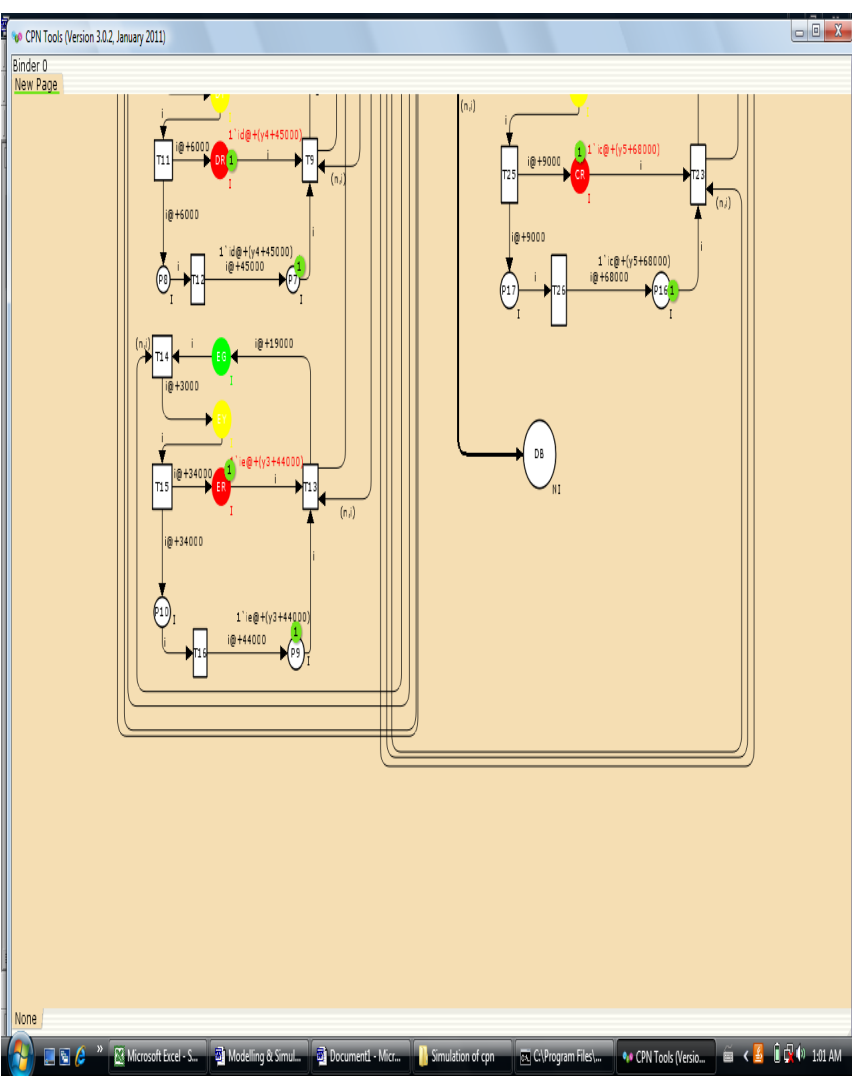

Fig 1.5: Lower part of the developed TCPN model of the T-type junction

Simulation of the developed TCPN Model: The developed Timed Coloured Petri Net Model of the Ttype Junction is simulated via CPN tools. CPN Tools is a tool suite for editing, simulation and performance analysis of CPN models. The user of CPN Tools works directly on the graphical representation of the TCPN model. The graphical user interface (GUI) of CPN Tools has no conventional menu bars and pulldown menus, but is based on interaction techniques such as tool palettes and marking menus. For this simulation work, the average time interval of vehicles entering an intersection with respect to a particular stream during green phase of each cycle is required by the CPN tools. Through the use of detectors, the data is obtained at the T-type junction under consideration. Precisely, the data is collected for ten timing plan cycles in one day. The average time interval of vehicles for each stream is approximated and shown in Table 1.5.

\section{DISCUSSION OF THE RESULTS}

Figure 1.6 compares the simulation data with the real traffic data, for each vehicle stream, based on the number of vehicles passing through the T-junction under consideration. There are ten columns each represents a cycle (i.e. column 1 represents cycle 1 , 
column 2 represents cycle 2 etc) in each vehicle stream. By considering stream DR, the columns of DR-simul in figure 1.6 are the simulated data of the stream DR. The first, second, third and tenth column is the vehicle number (i.e., 2, 3, 7 and 15) of cycle 1, 2,3 and 10 respectively which was simulated by the developed TCPN model. The model is with average time interval of vehicles (i.e. 2376, 15517, 6716, 3154 milliseconds). Likewise, the columns of DR-Real data in Figure 1.6 show the real measurement data from the T-type junction under consideration. It is obvious that the Figure 1.6 shows that the simulated results are almost consistent with the real traffic data obtained. The slight differences are due to modeling features such as the presence of pedestrians and motorcycles that are not modeled in the developed TCPN

model.

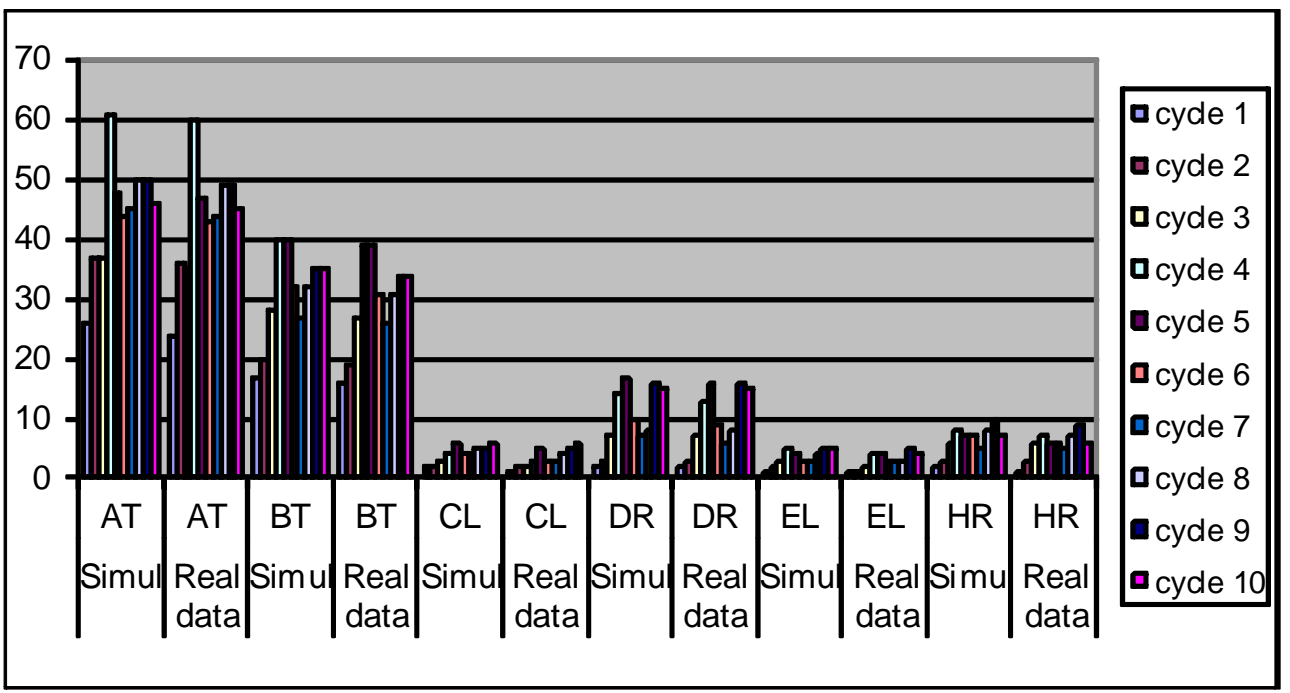

Fig 1.6: Comparison of Simulated and real traffic data for each stream of vehicle

\section{CONCLUSION}

This paper has employed a Timed Coloured Petri Net (TCPN) formalism to model and simulate a multiphase traffic light controlled junction with an associated fixed timing plan using T-type junction located in Federal Capital Territory, Abuja, Nigeria, as a case study. The development of the Timed Coloured Petri Net model is limited to fixed time control strategy as traffic is currently ruled in the considered intersection following the signal timing plan shown in Table 1.2. Also, the introduction of modelling features such as presence of pedestrians and motorcycles at the aforementioned junction are not considered. However, the developed Timed Coloured Petri Net (TCPN) model of the T-type junction could help in studying and improving traffic flow for a platoon of vehicles based on the knowledge acquired from the simulation. Also, the result of this work could serve as a guide in setting real-time actuated signal controller for the modelled scenarios or other related phenomenon.

\section{REFERENCES}

Dotoli, M., Fanti, M. P. and Meloni, C. (2003): "Real time optimization of traffic signal control: application to coordinated intersections", In Proc. IEEE Int. Conf. on Systems, Man and Cybernetics, Washington, pp. 3288-3295.

Gabard, J. F. (1991): "Car following models", In Concise encyclopaedia of traffic and transportation systems, pp. 65-68, M. Papageorgiou (Ed.), Oxford: Pergamon Press.

Ganiyu, R. A., Omodiora, E. O., Olabiyisi, S. O., Arulogun, O. T. and Okediran, O. O. (2011): "The underlying concepts of Coloured Petri Net (CPN) and Timed Coloured Petri Net (TCPN) models through illustrative example", Accepted Manuscript for Publication in International Journal of Physical Science, Paper No: ASCN/2011/012, African University Journal Series, Accra, Ghana.

Huang, Y. S. and Chung, T. H. (2008): "Modeling and Analysis of Urban Traffic Lights Control Systems Using Timed CP-nets", Journal of Information Science and Engineering, Vol. 24, pp. 875-890. 
Jensen, K. (1993): "Coloured Petri Nets with Time Stamps", Computer Science Department, Aarhus University, Denmark.

Jensen, K. (1994): "An Introduction to the Theoretical Aspects of Coloured Petri Nets", A Decade of Concurrency, Lecture Notes in Computer Science, Springer-Verlag, Vol. 803, pp. 230-272.

Jensen, K., Kristensen, L. M. and Wells, L. (2007): "Coloured Petri Nets and CPN Tools for modelling and validation of concurrent systems", International Journal on Software Tools for Technology Transfer, Vol. 9, No. 3-4, pp. 213-254.

Kutil, M., Hanzalek, Z. and Cervin, A. (2006): "Balancing the waiting times in a simple traffic intersection model", In 11th IFAC Symposium on Control in Transportation Systems.
Murata, T. (1989): "Petri Nets: Properties, Analysis and Applications", Proceedings of the IEEE, Vol. 77, No. 4, pp. 541-580.

Patel, M. and Ranganathan, N. (2001): "IDUTC: An Intelligent Decision-Making System for Urban TrafficControl Applications", IEEE Trans. on Vehicular Technology, Vol. 50, pp. 816-829.

Peterson, J.L. (1981): "Petri Net Theory and the Modeling of Systems", Prentice Hall, Englewood Cliffs, New Jersey.

Wey, W. M. (2000): "Model formulation and solution algorithm of traffic signal control in an urban network", Computers, environment and urban systems, Vol. 24, pp. 355-377. 